\title{
MULTIPLE STARS AS A RESULT OF THE DYNAMICAL EVOLUTION OF SMALL STELLAR SYSTEMS
}

\author{
Ávram Hayli
}

Université de Besançon

\section{INTRODUGTION}

Some double, triple or quadruple stars could be the final stage of the dynamical evolution of small galactic clusters. This evolution is nothing but a redistribution of the kinetic and mutual potential energy of the stars. It has two main effects: the escape of stars from the cluster and the formation of tight subsystems.

\section{NUMERIGAL SIMULATIONS OF SMALL CLUSTERS}

It is known that stars are often born in groups. If we ignore the dynamics of the gas and its effects on the motion of the stars and if we consider only the positions and velocities of the latter under their mutual attraction, one of the following situations occurs.

1) The sum of the kinetic and mutual potential energy of the stars is positive and the star system is of the association type. Such a system will be disrupted in a time of the order of a few million years.

2) The total energy is negative and the system is of the galactic cluster type. Things are a bit more complicated in a general galactic field but can be handled with no special difficulties.

Numerical simulations of galactic clusters of 15 to 50 stars revolving on circular orbits about the galactic center at the distance of the sun were performed. The density at the beginning was of the order of $10 \mathscr{H} \omega_{\odot c^{-3}}$ that is about 100 times larger than the critical value of disruption. At $t=0$ stars were distributed uniformly inside a sphere of radius
1 pc. The velocity distribution was uniform and the virial coefficient equal to 1 . The condition on this coefficient is not restrictive as simulations have shown that a cluster with negative total energy has an immediate tendency to adjust its dimensions and the velocities of its stars so that the virial relation is fulfilled.

In the course of time the more or less close encounters between the stars give to some of them enough energy to leave the cluster. On the other hand the tidal effect of the galactic field produces a continuous evaporation of the stars. Stars which escape usually carry with them little energy so that the total energy of the cluster remains approximately the same but is distributed among fewer stars.

For the poorest systems which have been followed through simulations, that is for systems starting with 15 stars, the final result was always the following. Almost all the stars leave the systems as the result either of the encounters or the ticlal force so that the cluster ends as a system of two, threc or, at most, four stars. When the stars have different masses, the ones involved in the final binary, triple or quadruple system, contain always the most, massive star. It happens also sometimes that a binary star which is formed early in the history of the evolution of the cluster is ejected after a close encounter with a heavier member of the system. In that case the remaining cluster continues its evolution as described previously.

\section{RESULTS}

The following table (Table 1) gives some results on simulations for clusters of 15 stars with dif- 
TABLE 1

\begin{tabular}{rrrrll}
\hline & $\begin{array}{c}\mathrm{t}_{\max } \\
\left(10^{8} \mathrm{yr}\right)\end{array}$ & $\mathrm{M}_{\mathrm{b}}, \mathrm{M}_{\mathrm{b}}^{\prime}$ & $\begin{array}{c}\mathrm{t}_{\mathrm{f}} \\
\left(10^{\circ} \mathrm{yr}\right)\end{array}$ & $\mathrm{N}_{\mathrm{r}}$ & $\begin{array}{c}\mathrm{d}_{\min } \\
(\mathrm{AU})\end{array}$ \\
\hline 1 & 255 & 8,4 & 105 & $4(8,4,2,2)$ & 2800 \\
2 & 125 & 8,2 & 48 & $4(8,4,2,1)$ & 1000 \\
3 & 275 & 8,2 & 163 & $3(8,4,2)$ & 2000 \\
4 & 55 & 8,4 & 16 & $3(8,4,1)$ & 2000 \\
5 & 120 & 8,2 & 78 & $3(8,2,2)$ & 1000 \\
6 & 150 & 8,4 & 38 & $2(8,4)$ & 1500 \\
\hline
\end{tabular}

ferent masses. Each cluster had one star of $8 \mathcal{M}_{\odot}, 2$ stars of $4 M_{\odot}, 4$ stars of $2 M_{\odot}$ and 8 stars of $19 \%_{0}$. Each cluster has been followed during the time $t_{\max }, M_{b}$ and $M_{b}^{\prime}$ are the masses of the two closest stars to each other between times $t_{f}$ and $t_{\max }$. $\mathrm{N}_{r}$ is the multiplicity of the final subsystem. Together with $\mathrm{N}_{\mathrm{r}}$ are given, in parentheses, the masses of the components: $d_{m i n}$ is the shortest distance between the components.

Minimum separations in this example are of the order of $1500 \mathrm{AU}$. In some casses $d_{m I n}$ as small as $500 \mathrm{AU}$ were observed but this was an absolute minimum. However it is not excluded that smaller separations could be reached through dynamical evolution. Some more details are given in Hayli (1971). An extensive study of this question is in progress.

\section{REFERENCES}

Hayli, A. 1971, Ap. and Space Sci., 13, 309. 